\title{
Bioequivalence and Pharmacokinetic Profiles of Agomelatine 25-mg Tablets in Healthy Chinese Subjects: A Four-Way Replicate Crossover Study Demonstrating High Intra- and Inter-Individual Variations
}

\author{
Cuiyun Li, ${ }^{a, \#}$ Jing Xu,,${ }^{b, \#}$ Yan Zheng, ${ }^{c}$ Guiling Chen,${ }^{a}$ Jianmeng Wang, ${ }^{c}$ Liying Ma,${ }^{d}$ \\ Yan Qiao, ${ }^{e}$ Junqi Niu, ${ }^{f}$ Min Wu, ${ }^{a}$ Hong Zhang, ${ }^{a}$ Xiaojiiao Li, ${ }^{a}$ Hong Chen, ${ }^{a}$ Xiaoxue Zhu, ${ }^{a}$ \\ Chengjiao Liu, ${ }^{a}$ and Yanhua Ding*,a \\ ${ }^{a}$ Phase I Clinical Unit, China-Frontage USA, The First Hospital of Jilin University; Changchun 130000, China: \\ ${ }^{b}$ Department of Neurology, The First Hospital of Jilin University; Changchun 130000, China: ${ }^{c}$ Department of \\ Geriatrics, The First Hospital of Jilin University; Changchun 130000, China: ${ }^{d}$ The Second Hospital of Jilin; \\ Jilin 132000, China: ${ }^{e}$ Department of Clinical Laboratory, The Second Hospital of Jilin; Jilin 132000, China: and \\ ${ }^{f}$ Department of Hepatology, The First Hospital of Jilin University; Changchun 130000, China. \\ Received November 7, 2016; accepted April 3, 2017; advance publication released online April 8, 2017
}

The present study was designed to assess the bioequivalence of two agomelatine formulations (25-mg tablets) in healthy Chinese male subjects. This single-dose, open-label, randomized, four-way replicate study with a 1-week washout period was conducted in 60 healthy Chinese male volunteers under fasting conditions. Blood samples were collected over a 12-h period after a single dose of the 25-mg agomelatine test (T) formulation or a reference ( $R$ ) formulation, and the drug concentrations were assayed by liquid chromatography tandem mass spectrometry (LC-MS/MS). Pharmacokinetic parameters were calculated using a noncompartmental model. Bioequivalence between the formulations was assessed. Tolerability and safety were monitored by physical examination, electrocardiogram (12-lead ECG), clinical laboratory tests, and adverse events (AEs). A total of 56 out of 60 subjects completed the study. No AEs were observed. The values of maximum plasma concentration $\left(C_{\max }\right)$, maximum concentration $\left(T_{\max }\right)$, area under curve $(A U C)_{0-t}$ and $t_{1 / 2}$ were $12.032 \mathrm{ng} / \mathrm{mL}, 0.658 \mathrm{~h}, 12.637 \mathrm{ng} \cdot \mathrm{h} / \mathrm{mL}$, and $0.813 \mathrm{~h}$, respectively, for the test formulation, and $10.891 \mathrm{ng} / \mathrm{mL}$, $0.709 \mathrm{~h}, 11.572 \mathrm{ng} \cdot \mathrm{h} / \mathrm{mL}$, and $0.96 \mathrm{~h}$, respectively, for the reference formulation. The intra-individual variability of $C_{\max }$ and $A U C_{0-t}$ were 78.3 and $61.8 \%$, respectively. The inter-individual coefficients of variance $(\mathrm{CVs})$ of $C_{\max }$ and $A U C_{0-t}$ were approximately $100 \%$. The $90 \%$ confidence intervals for the ratio of means for the log-transformed $C_{\max }(97.7-124.9 \%), A U C_{0-t}(98.2-118 \%)$, and $A U C_{0-\infty}(97.8-117.2 \%)$ were within the guideline range of bioequivalence (80-125\%). The test and reference formulations of agomelatine met the regulatory criteria for bioequivalence of the Chinese Food and Drug Administration. Significant intra-individual and inter-individual variations were found.

Key words agomelatine; bioequivalence; pharmacokinetic; intra-individual; inter-individual variation

Depression is one of the most common mental disorders worldwide. ${ }^{1)}$ As a complex and usually recurrent mental disorder, it poses a great burden on patients, family, and society. ${ }^{2}$ The treatment of depressive disorders is quite complex, and proper management of this disease is beneficial for society at large. The discovery of new drug targets or mechanisms of action for treating depression has attracted much attention as many patients are unresponsive to the available antidepressants and have difficulties in achieving remission. ${ }^{3)}$

Agomelatine, approved for marketing in the European Union and other countries, ${ }^{4,5)}$ is an agonist of melatonin MT1 and MT2 receptors and a weak 5-HT2C antagonist, but it has no activity on serotonin, norepinephrine (noradrenaline), or dopamine release. ${ }^{6,7)}$ A recent meta-analysis has suggested that it causes a greater reduction in symptom scores than the placebo and some other antidepressants; in addition, it has similar efficacy as standard drug treatment for depression. ${ }^{8)}$

Pharmacokinetic studies of agomelatine have demonstrated that it is rapidly $(80 \%)$ absorbed after oral administration, reaching the maximum plasma concentration $\left(C_{\max }\right)$ within $1-2 \mathrm{~h}$, with a half-life of $1-2 \mathrm{~h}$ in healthy subjects. ${ }^{9)}$ However,

\# These authors contributed equally to this work. its absolute oral bioavailability is low (3-4\%) with high interindividual variation (estimated to be $104 \%$ ), primarily due to its extensive first pass metabolism, ${ }^{10,11)}$ which brings a challenge to the bioequivalence study. ${ }^{12)}$ The apparent volume of distribution is $35 \mathrm{~L}$, and its protein binding rate is $95 \%{ }^{11)}$ Agomelatine is primarily metabolized by the hepatic CYP is enzymes in vivo. ${ }^{11)}$ Song et al. have demonstrated that a genetic polymorphism of CYP1A2 is associated with the marked inter-individual variation in agomelatine metabolism. ${ }^{13)} \mathrm{Be}-$ sides, the ingestion of high-fat food does not alter the absorption or the bioavailability of agomelatine. ${ }^{11)}$

To apply for marketing of a generic agomelatine formulation produced by Jinan Limin Pharmaceutical Co., Ltd., a bioequivalence study is required after approval for the clinical trial by the Chinese Food and Drug Administration (CFDA). There are few published pharmacokinetic data on agomelatine in healthy Chinese subjects. The present study was designed to generate more information on the pharmacokinetic characteristics of generic agomelatine in healthy Chinese subjects, in comparison with the brand name agomelatine. As agomelatine has marked intra-individual and inter-individual variations, a four-way replicate crossover study design was applied in the present bioequivalence study. 


\section{Experimental}

Drugs The Reference (R) formulation was Agomelatine 25-mg Tablet (Valdoxan ${ }^{\circledR}$ ), manufactured by Servier Industrie, Orléans, France (Lot Number: 933556; Expiry Date: March 2016). The Test ( $\mathrm{T})$ formulation was Agomelatine 25-mg, Tablet manufactured by Jinan Limin Pharmaceutical Co., Ltd., Jinan, China (Lot number: 140303; Expiry Date: February 2016). Both formulations were provided by Jinan Limin Pharmaceutical Co., Ltd.

Ethics Statement The test drug was first approved in a clinical trial by the CFDA on January 29, 2014 (registration number: 2014L00220). The study protocol was reviewed and approved by the Ethics Committee of The First Hospital of Jilin University, Changchun, China (approval number: 12066), on April 3, 2014. The study was conducted according to the Declaration of Helsinki and the Guidelines for Good Clinical Practice, and a written informed consent was obtained from each of the participants prior to study initiation. In addition, the clinical trial procedure abided by the Good Clinical Practice of the International Conference on Harmonization (ICHGCP)..$^{14)}$

Subjects Eligible subjects were healthy males between the ages of 18 and 40 years old who were at least $50 \mathrm{~kg}$ with a body mass index (BMI) between 19 and $24 \mathrm{~kg} / \mathrm{m}^{2}$. The subjects were enrolled into the study as determined by a complete medical examination, including medical history, physical examination, 12-lead electrocardiogram (ECG), chemistry, hematology, urinalysis, hepatitis $\mathrm{B}$ and $\mathrm{C}$, and human immunodeficiency virus (HIV) diagnostic profiles.

Subjects with a history of significant use of alcohol, cigarettes, or abusive drugs within 1 year of screening, or allergic reactions to any drug were excluded. Administration of any drugs within $14 \mathrm{~d}$ prior to the initial dose was not allowed. Subjects were instructed to abstain from consuming alcoholic, caffeine-, or xanthine-containing food or beverages from $48 \mathrm{~h}$ prior to dosing.

In the present study, 111 subjects were screened, including 51 who were excluded and 60 who were enrolled. A total of 56 subjects completed all the sessions of the study and were included in the analysis. Four subjects withdrew from the study due to personal reasons.

Study Design and Drug Administration The study was a single-dose, randomized, open-label, four-way replicate (i.e., $\mathrm{T}, \mathrm{R}, \mathrm{T}, \mathrm{R}$ or $\mathrm{R}, \mathrm{T}, \mathrm{R}, \mathrm{T}$ ) study. Before drug administration, the volunteers were randomly divided into two groups (A, B) by using DAS3.2.5 software. Group A was given the drugs in the sequence of $\mathrm{T}, \mathrm{R}, \mathrm{T}$, and $\mathrm{R}$ for the four sessions; and the sequence for Group B was R, T, R, and T. There was a 7-d washout period between the sessions. After an overnight fast of at least $10 \mathrm{~h}$, the subjects received a single oral dose of the $\mathrm{R}$ or $\mathrm{T}$ formulation of Agomelatine Tablets $(25 \mathrm{mg})$ with $250 \mathrm{~mL}$ of water in a seated position, followed by a mouth check to assess administration compliance.

Blood Sample Collection and Plasma Preparation The blood samples were collected at 0 (within $30 \mathrm{~min}$ prior to dosing) and $0.17(10 \mathrm{~min}), 0.33(20 \mathrm{~min}), 0.5,0.75,1.0,1.25,1.5$, $2.0,2.5,3.0,4.0,5.0,6.0,8.0,10.0$, and $12.0 \mathrm{~h}$ post dosing and placed into chilled blood collection tubes containing heparin sodium. The samples were then centrifuged at $3500 \mathrm{rpm}$ at $4^{\circ} \mathrm{C}$ for $10 \mathrm{~min}$ to separate the plasma; the plasma samples were stored at $-70^{\circ} \mathrm{C}$ until analysis. At the end of the study, one of the two frozen plasma aliquots, together with sufficient dry ice, were sent from the clinical facility to the bioanalytical facility. The temperature was monitored to ensure that the samples were in a frozen state during the shipping. The other set of aliquots was kept in the clinical facility for backup.

Analysis of Plasma Samples The plasma concentrations of agomelatine were detected by Anhui Wanbang Medical Technology Co., Ltd., Hefei, China. An original liquid chromatography tandem mass spectrometry (LC-MS/MS) method was developed and validated for quantification of agomelatine in human plasma. The plasma sample was thawed at room temperature, and $200 \mu \mathrm{L}$ of the plasma was mixed with $20 \mu \mathrm{L}$ of internal standard working solution $(100 \mathrm{ng} / \mathrm{mL}$ diazepam purchased from The National Institute for the Control of Pharmaceutical and Biological Products, Beijing, China). To the mixture, $4 \mathrm{~mL}$ of ethyl acetate was added, and the sample was vortexed for $1 \mathrm{~min}$. The mixture was then centrifuged for $10 \mathrm{~min}$ at $3000 \mathrm{rpm}$, and the upper organic layer was transferred into evaporation tubes. The samples were then evaporated under nitrogen at $40^{\circ} \mathrm{C}$ and reconstituted with $0.3 \mathrm{~mL}$ of mobile phase. A $20-\mu \mathrm{L}$ aliquot was injected onto the LC-MS/MS system.

Liquid chromatography was performed on an Agilent 1100 system equipped (Agilent Technologies Inc., Santa Clara, CA, U.S.A.) with an autosampler. The system was coupled to the API 3000 via an electrospray ionization interface for mass analysis and detection. A Phenomenex Luna C18 column $(2.0 \times 100 \mathrm{~mm}, 5 \mu \mathrm{m}$, Phenomenex Inc., Torrance, CA, U.S.A.) was used for chromatographic separation. The mobile phase was methanol- $0.1 \%$ formic acid $(80: 20, \mathrm{v} / \mathrm{v})$. The flow rate was $0.2 \mathrm{~mL} / \mathrm{min}$, and the column temperature was set at $40^{\circ} \mathrm{C}$.

The detection was performed with the mass spectrometer in positive electrospray ionization mode. The multiple reaction monitoring transitions were a mass to charge ratio of 244.0/185.0 for agomelatine and 285.1/154.0 for diazepam. The optimized instrumental parameters for mass spectral acquisition were as follows: collision gas at $10 \mathrm{psi}$, curtain gas at 10 psi, dwell time of $200 \mathrm{~ms}$, ion spray voltage of $4000 \mathrm{~V}$, and temperature of $400^{\circ} \mathrm{C}$.

According to the Guidelines for Good Laboratory Practice and CFDA guidelines, the bioanalytical process was validated in terms of linearity, precision, accuracy, specificity, selectivity, lower limit of quantitation (LLOQ), recovery, matrix effect, ionic suppression, and stability under different conditions. The method had good linearity $\left(r^{2} \geq 0.9966\right)$ and allowed the quantification of agomelatine in human plasma within the range of 0.05 to $20 \mathrm{ng} / \mathrm{mL}$, with the LLOQ being $0.05 \mathrm{ng} / \mathrm{mL}$. For the samples with concentrations of over $20 \mathrm{ng} / \mathrm{mL}$, the samples were diluted with blank human plasma in reasonable ratio before analysis. Meanwhile, the quality of control (QC) samples were diluted with the same ratio to validate the dilution integrity. No peak interfering with quantitation was observed throughout the validation process. The relative standard deviations of inter-day and intra-day precision were within $15 \%$ of the four QC levels $(0.125,1.5,8$, and $16 \mathrm{ng} / \mathrm{mL}$ ), and the deviation of accuracy was restrained from 85 to $115 \%$. The recoveries of agomelatine from plasma were between 87.97 and $95.10 \%$. No significant matrix effects were observed. The analyte was stable in human plasma after three freeze-thaw cycles, for $8 \mathrm{~h}$ at room temperature, for up to $50 \mathrm{~d}$ at $-20^{\circ} \mathrm{C}$, and for $22 \mathrm{~h}$ after processing for analysis. 
Pharmacokinetic, Bioequivalence, and Statistical Analyses The pharmacokinetic parameters were calculated individually for each subject from the plasma-time profile using a non-compartmental approach. The $C_{\max }$ and the time to reach maximum concentration $\left(T_{\max }\right)$ were obtained directly from the measured data; the half-life of drug elimination during the terminal phase $\left(t_{1 / 2}\right)$, the area under the plasma concentrationtime curve from 0 to the last measurable concentration (area under curve $\left.(A U C)_{0-t}\right)$, and the area under the plasma concentration-time curve from 0 to infinity $\left(A U C_{0-\infty}\right)$ were computed using DAS 3.2.5 software.

Statistical analysis of bioequivalence parameters was also carried out using DAS 3.2.5 software. For $\ln C_{\max }, \ln A U C_{0-t}$, and $\ln A U C_{0-\infty}$, variance analysis was used to assess the period, person, and product effects. Two/one-sided Student's $t$-tests for pharmacokinetic parameters $\left(\ln C_{\max }, \ln A U C_{0-t}\right.$, and $\left.\ln A U C_{0-\infty}\right)$ of agomelatine between the two formulations were conducted. Bioequivalence was assessed using a $90 \%$ confidence interval (CI) for the log-transformed bioequivalence parameters, within an acceptable range of $80-125 \%$. For variables such as height, weight, and age, standard descriptive analysis including mean and standard deviation (S.D.) were applied. These statistical parameters including coefficient of variance $(\mathrm{CV})$ were used to describe plasma concentrations at each time point and each of the pharmacokinetic parameters.

Evaluation for Tolerability and Safety The tolerability to the $\mathrm{T}$ and $\mathrm{R}$ formulations was assessed by monitoring of adverse events (AEs), vital signs (temperature, blood pressure, heart rate, and respiratory rate), and laboratory tests (chemistry, hematology, and urinalysis) from the screening period to the end of the study. ECGs and clinical laboratory tests were conducted before drug administration and at the completion of the study. The vital signs were measured at the time of screening and within $1 \mathrm{~h}$ before and 1, 3, 6, and $12 \mathrm{~h}$ after dosing.

\section{Results}

Characteristics of the Subjects In the bioequivalence study, 60 subjects were enrolled, and 56 of them completed all the sessions of the study and were therefore included in the pharmacokinetic and statistical analyses. Four subjects (two subjects prior to dosing in session II and two in session IV)

Table 1. Demographic Data of the Subjects Who Completed the Bioequivalence Study

\begin{tabular}{lc}
\hline \hline Variable & Value $(n=56)$ \\
\hline Age (years) & $23.23 \pm 2.56$ \\
Mean \pm S.D. & $20-29$ \\
Range & \\
Body weight $(\mathrm{kg})$ & $66.9 \pm 7$ \\
Mean \pm S.D. & $52-80$ \\
Range & $175 \pm 5$ \\
Body height $(\mathrm{cm})$ & $164.5-184$ \\
Mean \pm S.D. & \\
Range & $21.88 \pm 1.70$ \\
BMI $\left(\mathrm{kg} / \mathrm{m}^{2}\right)$ & $19.05-24.00$ \\
Mean \pm S.D. & \\
Range & $53(94.6 \%)$ \\
Race & $3(5.4 \%)$ \\
Han & \\
Others &
\end{tabular}

had withdrawn from the study due to personal reasons that were unrelated to the administration of test or reference drugs. The demographic data of the 56 subjects are summarized in Table 1.

Tolerability and Safety The safety of both formulations was evaluated through the assessment of AEs, laboratory examinations, and vital signs. Both clinical and laboratory parameters of all subjects showed no clinically significant changes in any of the subjects in any sessions. Moreover, no AEs were observed during the study in any of the subjects in any sessions.

Pharmacokinetics The mean (S.D.) plasma concentrations of the $\mathrm{R}$ and $\mathrm{T}$ formulations versus time profiles are presented in Figs. 1a and b, respectively (GraghPad Prism 5

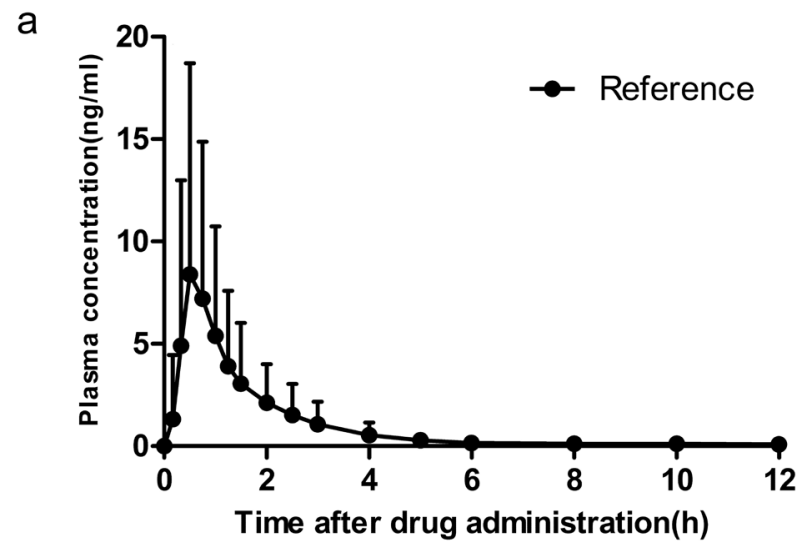

b

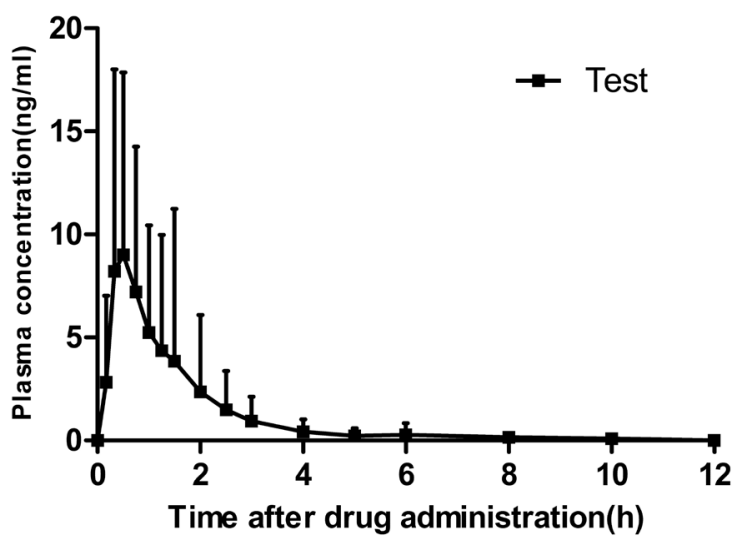

C

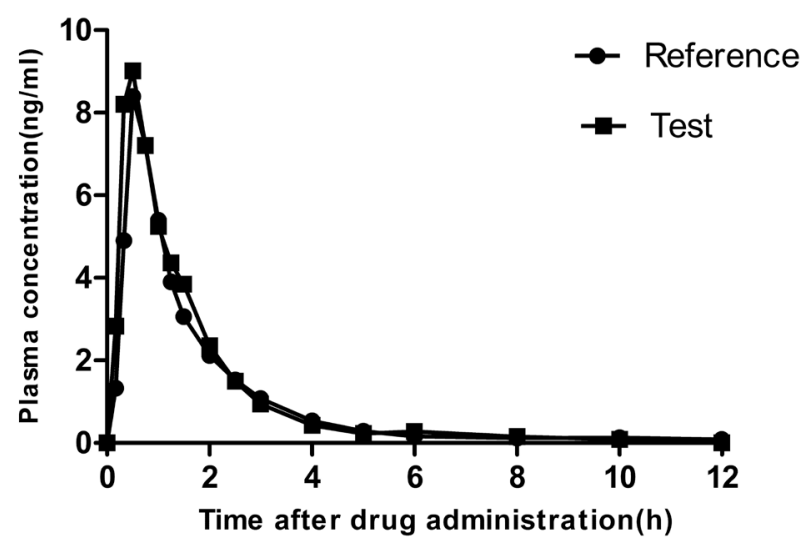

Fig. 1. Mean Plasma Agomelatine Concentrations Obtained after a Single 25-mg Dose Administration of the R or T Formulation

$\mathrm{a}, \mathrm{R}$ formulation; $\mathrm{b}, \mathrm{T}$ formulation; $\mathrm{c}$, Comparison of the $\mathrm{R}$ and $\mathrm{T}$ formulations. 
Table 2. Pharmacokinetic Parameters of the T and R Formulations

\begin{tabular}{|c|c|c|c|c|c|c|}
\hline \multirow{2}{*}{ Pharmacokinetic parameters } & \multicolumn{3}{|c|}{$\mathrm{T}$} & \multicolumn{3}{|c|}{$\mathrm{R}$} \\
\hline & Mean (range) & S.D. & $\mathrm{CV}$ & Mean (range) & S.D. & $\mathrm{CV}$ \\
\hline$\left.C_{\max }(\mathrm{ng} / \mathrm{mL})^{a}\right)$ & $12.032(1.05-75.4)$ & 12.219 & 100.9 & $10.891(1.06-62.1)$ & 11.043 & 102.3 \\
\hline$T_{\max }(\mathrm{h})$ & 0.658 & 0.492 & 75.4 & 0.709 & 0.464 & 65 \\
\hline$t_{1 / 2}(\mathrm{~h})$ & 0.813 & 0.359 & 44.1 & 0.960 & 0.847 & 87.6 \\
\hline$A U C_{0-t}{ }^{a)}(\mathrm{ng} \cdot \mathrm{h} / \mathrm{mL})$ & $12.637(1.63-108.0)$ & 12.868 & 101.6 & $11.572(1.37-65.0)$ & 10.343 & 89.6 \\
\hline$A U C_{0-\infty}{ }^{a)}(\mathrm{ng} \cdot \mathrm{h} / \mathrm{mL})$ & $12.795(1.78-108.0)$ & 12.886 & 100.7 & $11.71(1.40-65.5)$ & 10.363 & 88.5 \\
\hline $\mathrm{F}^{b)}$ & 108.3 & 30.8 & 28.418 & & & \\
\hline $\mathrm{F} 1^{c)}$ & 107.4 & 28.9 & 26.864 & & & \\
\hline
\end{tabular}

a) The data are expressed as mean (min-max). $b$ ) Relative bioavailability as calculated by $A U C_{0-t^{*}} c$ ) Relative bioavailability as calculated by $A U C_{0-\infty}$.

Table 3. Ratios (T/R) and $90 \%$ CIs Following the Administration of Agomelatine Tablets

\begin{tabular}{llll}
\hline \hline Parameter & $\mathrm{T}$ & $\mathrm{R}$ & $\mathrm{T} / \mathrm{R}(\%)$ \\
\hline$\left.C_{\max }(\mathrm{ng} / \mathrm{mL})^{a}\right)$ & 8.29 & 7.504 & 110.5 \\
$A U C_{0-t}(\mathrm{ng} \cdot \mathrm{h} / \mathrm{mL})^{a)}$ & 9.156 & 8.507 & 107.6 \\
$A U C_{0-\infty}(\mathrm{ng} \cdot \mathrm{h} / \mathrm{mL})^{a)}$ & 9.314 & 8.702 & 107.0 \\
\hline
\end{tabular}

a) Geometric average values.

software). The curves are superimposed on each other (Fig. 1c). The pharmacokinetic parameters of agomelatine for the $\mathrm{T}$ and $\mathrm{R}$ formulations appear in Table 2.

The $C_{\max }$ and $A U C_{0-t}$ values of the $\mathrm{T}$ formulation were slightly higher than those of the $\mathrm{R}$ formulation. In terms of absorption, the ratios (T/R) of both $A U C_{0-t}$ and $A U C_{0-\infty}$ were very close to $100 \%$. Based on the plasma concentrations, the mean relative bioavailability of the $\mathrm{T}$ formulation was $108.3 \%$, compared with that of the $\mathrm{R}$ formulation.

Specifically, the $C_{\max }$ of all the subjects ranged from 1.05 to $75.4 \mathrm{ng} / \mathrm{mL}$ for the $\mathrm{T}$ formulation; and it ranged from 1.06 to $62.1 \mathrm{ng} / \mathrm{mL}$ for the $\mathrm{R}$ formulation. The ranges of the $A U C$ for the $\mathrm{T}$ and $\mathrm{R}$ formulations were $1.78-108.0 \mathrm{ng} \cdot \mathrm{h} / \mathrm{mL}$ and $1.40-65.5 \mathrm{ng} \cdot \mathrm{h} / \mathrm{mL}$, respectively. The intra-individual variability of $C_{\max }$ and $A U C_{0-t}$ were 78.3 and $61.8 \%$, respectively. The inter-individual CVs of $C_{\max }$ and $A U C_{0-t}$ were approximately 100\% (Table 2).

Bioequivalence The geometric mean ratios (T/R) and $90 \%$ CIs for the primary pharmacokinetic parameters $\left(C_{\max }\right.$, $A U C_{0-t}$, and $A U C_{0-\infty}$ ) of the two agomelatine formulations are presented in Table 3. The data demonstrated that the ratios (T/R) and corresponding $90 \%$ CIs of the relative $C_{\max }, A U C_{0-t}$, and $A U C_{0-\infty}$ of the two formulations ranged from 80 to $125 \%$ under fasting conditions. The ANOVA results revealed that different periods or drug treatments had no statistically significant effects on $C_{\max }, A U C_{0-t}$, or $A U C_{0-\infty}(p>0.05)$. However, there was a significant effect of the subject on these parameters $(p<0.05)$.

\section{Discussion}

To the best of our knowledge, the present study was the first bioequivalence study of agomelatine conducted in healthy Chinese male subjects. The study aimed to assess the bioequivalence of a $25-\mathrm{mg}$ agomelatine tablet with the brand name of Valdoxan ${ }^{\circledR}$ and the test formulation indigenously manufactured by Jinan Limin Pharmaceutical Co., Ltd.

Unlike many other antidepressants that inhibit neuronal reuptake of monoamines, agomelatine brings a new concept into the treatment of depression by combining its melatonin MT1 and MT2 agonist properties with a serotonin 5-HT2C antagonist effect. It has better efficacy than the placebo and an equal efficacy to other antidepressants. It also has better tolerability and safety than other antidepressants. ${ }^{15)}$ In the present study, no AEs occurred in any of the subjects, and laboratory examinations showed no clinically significant changes in the test parameters, demonstrating that $25-\mathrm{mg}$ agomelatine was well tolerated in Chinese subjects for both the $\mathrm{T}$ and $\mathrm{R}$ formulations.

For a highly variable drug, it may be difficult to demonstrate the bioequivalence between the generic product and the reference product in a two-way crossover study. ${ }^{15)}$ Several measures should be taken to obtain adequate statistical power to meet bioequivalence limits, including increasing the sample size and using a four-way $(2 \times 4)$ replicated crossover design. Therefore, the present bioequivalence study was designed as a single-dose, randomized, open-label, four-way replicated crossover study. Both the European Medicines Agency (EMA) and the U.S. Food and Drug Administration (FDA) recommend a four-way $(2 \times 4)$ replicated crossover design be used in the assessment of bioequivalence for highly variable drugs because the usual $2 \times 2$ crossover design may not provide enough power to establish bioequivalence. ${ }^{16)}$ Otherwise, a larger number of subjects and a higher cost would be necessary. Estimation of the sample size is another critical issue that needs to be addressed to ensure adequate power to get significant results. In addition, it needs to be calculated based on the intraindividual $\mathrm{CV}$ of pharmacokinetic parameters $\left(C_{\max }\right.$ and $\left.A U C\right)$ of reference products. According to the available agomelatine pilot data, the sample size in the present study was estimated by referencing the literature by Chow et al. ${ }^{17)}$

For determination of agomelatine in human plasma, an original LC-MS/MS method was developed and validated. Compared to the reported methods, ${ }^{18,19)}$ it was less time-consuming and had a wider range of quantification $(0.05-20 \mathrm{ng} / \mathrm{mL})$. The 
Table 4. Mean Pharmacokinetic Parameters of Agomelatine (25 mg) in Healthy Caucasian Subjects and Chinese Subjects

\begin{tabular}{lccc}
\hline \hline & \multirow{2}{*}{$\begin{array}{c}\text { Caucasian subjects } \\
(n=8)\end{array}$} & \multicolumn{2}{c}{ Chinese subjects $(n=56)$} \\
\cline { 3 - 4 } & & $\mathrm{R}$ & $\mathrm{T}$ \\
\hline$C_{\max }(\mathrm{ng} / \mathrm{mL})$ & $3.0 \pm 2.8$ & $10.795 \pm 11.043$ & $12.111 \pm 12.219$ \\
$t_{1 / 2}(\mathrm{~h})$ & $0.9 \pm 0.4$ & $0.966 \pm 0.847$ & $0.814 \pm 0.359$ \\
$A U C(\mathrm{ng} \cdot \mathrm{h} / \mathrm{mL})$ & $4.9 \pm 5.6$ & $11.71 \pm 10.363$ & $12.795 \pm 12.886$ \\
\hline
\end{tabular}

sampling scheme design was considered adequate for the determination of pharmacokinetic parameters with sufficient duration to fully assess the absorption and elimination phases of agomelatine. ${ }^{11)}$ The ratios of $A U C_{0-t}$ and $A U C_{0-\infty}$ for both the $\mathrm{T}$ and $\mathrm{R}$ formulations were $>80 \%$. The elimination $t_{1 / 2}$ for agomelatine was approximately $0.9 \mathrm{~h}$. Therefore, the washout period of 1 week was sufficient for complete elimination of the drug before subsequent administration, and no pre-dose concentrations were observed in sessions 2,3 , or 4 , demonstrating that the present study design was proper to meet the goal of the study.

Of note, it is reported that hepatic adverse reactions including elevation of alanine aminotransferase and/or aspartate aminotransferase is observed in $1.1 \%(25 \mathrm{mg})$ and $3.1 \%(50 \mathrm{mg})$ of Caucasian patients treated with agomelatine, ${ }^{20)}$ suggesting that the adverse reaction may be dose-dependent.

The pharmacokinetic parameters of agomelatine $(25 \mathrm{mg})$ under the fasting state in the present study were quite different from those obtained in Caucasians. ${ }^{11)}$ The $A U C$ values were approximately two-fold higher than those in Caucasians (Table 4), probably due to a less obvious first-pass effect; while the $t_{1 / 2}$ values of the two ethnic groups were similar. ${ }^{11)}$ In addition, high intra- (61.8\%) and inter-individual (89.4\%) variations of drug exposure were found in the present study. Hence, one should pay close attention to the clinical use of the drug, and further studies are needed to determine if a high incidence of transaminase elevation appears with $50 \mathrm{mg}$ dose use in Chinese patients.

The inter- and intra-individual variations of agomelatine have been investigated among different ethnic groups such as Caucasians and Chinese. ${ }^{3,21,22)}$ In Caucasians, the interindividual variability of the $A U C$ after the oral administration of agomelatine varied in different reports $(100-150 \% \mathrm{CV}){ }^{11)}$ It has also been reported that the intra-individual $\mathrm{CVs}$ of $C_{\max }$ and $A U C_{0-t}$ are 84.34 and $49.61 \%$ in healthy Chinese male subjects and that the inter-individual $\mathrm{CVs}$ of $C_{\max }$ and $A U C_{0-t}$ are 102.20 and $131.74 \%$, respectively (12 male subjects). ${ }^{22)}$ In the present study, large intra- and inter-individual variations of the pharmacokinetic parameters of agomelatine $\left(C_{\max }\right.$ from 1.06 to $62.1 \mathrm{ng} / \mathrm{mL}, A U C$ from 1.40 to $65.5 \mathrm{ng} \cdot \mathrm{h} / \mathrm{mL}$ ) were also observed; the intra-individual variability of $C_{\max }$ and $A U C_{0-t}$ were 78.3 and $61.8 \%$, respectively, and the inter-individual CVs of $C_{\max }$ and $A U C_{0-t}$ were both around $100 \%$.

Agomelatine is primarily metabolized by CYP1A2 (90\%) and by CYP2C9 and CYP2C19 (10\%). As CYP1A2 plays a critical role in the liver metabolism, its activity variation may result in the inter-individual variability of agomelatine. In fact, many factors could induce or inhibit the CYP1A2 activity. Any factors affecting CYP1A2 activity such as tabacco, coffee, cabbages, and grapefruit juice may directly change the pharmacokinetics of agomelatine and increase high intra-indi- vidual variability. Several CYP1A2 single nucleotide polymorphism (SNPs, such as srs762551, rs2470890, and rs2472304) have been reported to be involved in the marked variability in metabolism and drug concentrations. ${ }^{13)}$ This may also provide an explanation to the different pharmacokinetic profiles in different races. Agomelatine is extensively distributed throughout the body, which might also result in high inter-individual variability. ${ }^{23)}$ However, as the drug has a wide therapeutic window with an excellent safety profile, the high inter- and intra-individual variability may not remarkably affect its clinical use.

\section{Conclusion}

The generic and brand name formulations of agomelatine $(25 \mathrm{mg})$ met the regulatory criteria for bioequivalence, according to the CFDA guidelines. Both formulations were well tolerated by the enrolled healthy Chinese male volunteers. The intra-individual and inter-individual blood concentrations of agomelatine were highly variable. The four-way replicate crossover study was successfully applied in the bioequivalence assessment of agomelatine, demonstrating high intra- and inter-individual variations. Additionally, the $A U C$ values of agomelatine in Chinese subjects were found to be approximately two-fold higher than those in Caucasians, as reported previously. These results will ensure better use of this drug in Chinese populations.

Acknowledgment We would like to thank all the healthy volunteers for their participation in this study.

Conflict of Interest The authors declare no conflict of interest.

\section{References}

1) Kessler R. C., Aguilar-Gaxiola S., Alonso J., Chatterji S., Lee S., Ormel J., Ustün T. B., Wang P. S., Epidemiol. Psichiatr. Soc., 18, 23-33 (2009)

2) Hickie I., BMJ, 335, 329 (2007).

3) Plesničar B. K., Prefer. Adherence, 2, 603-612 (2014).

4) European Medicines Agency, "Valdoxan (agomelatine).": ‘www.ema. europa.eu/ema/index.jsp?curl=pages/medicines/human $/$ medicines/ 000915/human_med_001123.jsp\&mid=WC0b01ac058001d124>, cited 2012.

5) Hickie I. B., Rogers N. L., Lancet, 378, 621-631 (2011).

6) Racagni G., Riva M. A., Molteni R., Musazzi L., Calabrese F., Popoli M., Tardito D., World J. Biol. Psychiatry, 12, 574-587 (2011).

7) Sansone R. A., Sansone L. A., Innov. Clin. Neurosci, 8, 10-14 (2011).

8) Taylor D., Sparshatt A., Varma S., Olofinjana O., BMJ, 348 (mar19 5), g1888 (2014).

9) Buoli M., Mauri M. C., Altamura A. C., Expert Opin. Drug Metab. Toxicol., 10, 885-892 (2014).

10) Zhou Y., Chu W., Lei M., Li J., Du W., Zhao C., Int. J. Pharm., 473 250-258 (2014)

11) European Medicines Agency, "CHMP Assessment Report for Valdoxan.”: 〈http://www.ema.europa.eu/docs/en_GB/document_library/ EPAR__Public_assessment_report/human/000916/WC500038315. pdf), cited 2008.

12) Sánchez O M. P., Ocaña J., Carrasco J. L., Pharm. Stat., 10, 135142 (2011).

13) Song L., Du Q., Jiang X., Wang L., J. Clin. Pharm. Ther., 39, 204-209 (2014)

14) Dixon J. R. Jr., Qual. Assur., 6, 65-74 (1998).

15) Liu Y., Hu C., Liu G., Jia J., Yu C., Zhu J., Zheng Q., Zhang K. E., 
Agents. Chemother., 58, 6009-6015 (2014).

16) Uygungül E., Ayrik C., Narci H., Erdoğan S., Toker I., Demir F., Karaaslan U., Adv. Hematol., 2014, 369084 (2014).

17) Wang H., Chow S. C., J. Biopharm. Stat., 12, 295-309 (2002).

18) Wang X., Zhang D., Liu M., Zhao H., Du A., Meng L., Liu H., Chromatogr., 28, 218-222 (2014).

19) Patil S. R., Nerurkar K. K., Kalamkar A. M., Pukale V., Mangaonkar K. V., Pingale S. G., J. Mass Spectrom., 47, 23-28 (2012).
20) Goodwin G. M., Emsley R., Rembry S., Rouillon F., J. Clin. Psychiatry, 70, 1128-1137 (2009).

21) Pei Q., Wang Y., Hu Z. Y., Liu S. K., Tan H. Y., Guo C. X., Zhang R. R., Xiang Y. X., Huang J., Huang L., Yuan H., Yang G. P., PLoS ONE, 9, e109300 (2014).

22) Wang X. L., Du A. H., Zhang D., Meng L. J., Liu M., Zhang L. N., Zhao H. N., Liu H. C., Drug Res. (Stuttg.), 65, 552-554 (2015).

23) Owen R. T., Drugs Today (Barc.), 45, 599-608 (2009). 